\section{Processing of emotional faces in social phobia}

\section{Soren Rislov Staugaard, 1 \\ Nicole Kristjansen Rosenberg ${ }^{2}$}

1 Center on Autobiographical Memory

Research, Aarhus University; ${ }^{2}$ Clinic for

Anxiety Disorders, Aarhus University

Hospital, Aarhus, Denmark

\section{Abstract}

Previous research has found that individuals with social phobia differ from controls in their processing of emotional faces. For instance, people with social phobia show increased attention to briefly presented threatening faces. However, when exposure times are increased, the direction of this attentional bias is more unclear. Studies investigating eye movements have found both increased as well as decreased attention to threatening faces in socially anxious participants. The current study investigated eye movements to emotional faces in eight patients with social phobia and 34 controls. Three different tasks with different exposure durations were used, which allowed for an investigation of the time course of attention. At the early time interval, patients showed a complex pattern of both vigilance and avoidance of threatening faces. At the longest time interval, patients avoided the eyes of sad, disgust, and neutral faces more than controls, whereas there were no group differences for angry faces.

\section{Introduction}

As social beings, we encounter a multitude of faces every day and many of these will wear an emotional expression. Such expressions are potent social cues that hold information about the emotional and intentional state of people in our surroundings ${ }^{1-2}$ and that readily draw attention..$^{3-4}$ In a number of seminal studies $^{5-6}$ on recognition of emotional facial expressions in patients with bilateral damage to the amygdala, Adolphs and colleagues suggested that the amygdala is responsible for guiding attention towards those features of a human face that contain the emotional information (e.g., the eyes). Other studies have shown that patients with social phobia show an increased activation of the amygdala when confronted with emotional facial expressions. ${ }^{7}$ From a neurobiological perspective, hyperactivation of the amygdala may lead to an increased awareness of salient features of emotional human faces.

Cognitive theories of social anxiety predict that socially anxious individuals show increased vigilance for signs of social threat in their environment, such as a rejecting facial expression or a piercing gaze.8-9 This increased vigilance for threat - known as attentional bias - is hypothesized to be a causal or maintaining factor in social anxiety. Importantly, Clark and Wells ${ }^{8}$ suggest that socially anxious individuals should also show strategic avoidance of threatening cues, as an attempt to reduce the anxious feelings they may evoke. Combining these two propositions, Mogg, Bradley, de Bono, and Painter ${ }^{10}$ put forth a vigilance-avoidance theory, which predicts that initial automatic vigilance for threat is followed by later strategic avoidance.

\section{The dot-probe task}

Several studies using the dot-probe paradigm have shown that individuals with social phobia respond differently from healthy controls when shown emotional faces. ${ }^{11-14}$ In the dot-probe task, a pair of faces consisting of a neutral and an emotional expression is shown simultaneously on a monitor side by side or one above the other. Following a brief interval - typically $500 \mathrm{~ms}$ - the faces disappear from view and a small probe will appear in the position of either the neutral face or the emotional face. The participant's task is to respond to the probe as quickly as possible by pressing a button, and the reaction time can then be used as an indicator of which expression the participant was attending to.11 Dot-probe studies have shown both increased vigilance and avoidance in individuals with social phobia compared with controls, ${ }^{7}$ and it is possible that the dot-probe task has problems with either validity or reliability, or both.15-16 In favour of the task's validity, Mogg, Garner, and Bradley 17 found that the reaction time index significantly correlated with eye movements to the faces $(r=.35)$.

\section{Eye tracking}

Eye tracking allows researchers to directly track participants' eye movements across stimuli without having to rely on the interpretation of behavioural responses. Given its high temporal resolution, an eye tracker can provide detailed information about the time course of attention. In relation to social anxiety and processing of emotional faces in adult participants, eye tracking is starting to gain more widespread use. At the time of writing, nine studies from six research groups have been published, and four of these included patients with social phobia, ${ }^{18-21}$ while the rest relied on university students selected for high (HSA) and low (LSA) social anxiety.22-26

In the two studies by Horley, Williams, Gonsalvez, and Gordon,19-20 patients with
Correspondence: Søren Risløv Staugaard, Center on Autobiographical Memory Research, Department of Psychology and Behavioural Sciences, Aarhus University, DK-8000, Aarhus, Denmark.

Tel. +45.8942 .4955 - Fax: +45.8942 .4901$

E-mail: sorsta@psy.au.dk

Key words: attention, social phobia, eye movements, face processing, emotion.

Acknowledgements: the authors are very grateful to Peter Krøjgaard for valuable comments on earlier drafts of this manuscript, and to Louise Sørensen for her assistance in preparing the data. The eye tracker was purchased on a grant given to Peter Krøjgaard by The Danish Council for Independent Research (Humanities), grant no. 273-07-0009. Development of the MacBrain Face Stimulus Set was overseen by Nim Tottenham and supported by the John D. and Catherine T. MacArthur Foundation Research Network on Early Experience and Brain Development. Please contact Nim Tottenham at tott0006@tc.umn.edu for more information concerning the stimulus set.

Contributions: SRS, study design, data collection and analysis, first manuscript drafting; NKR, patient group clinically responsible, cooperation in manuscript drafting.

Received for publication: 31 May 2011.

Revision received: 6 July 2011.

Accepted for publication: 17 August 2011

This work is licensed under a Creative Commons Attribution NonCommercial 3.0 License (CC BYNC 3.0).

(C) Copyright S.R. Staugaard and N.K. Rosenberg, 2011 Licensee PAGEPress, Italy

Mental Illness 2011; 3:e5

doi:10.4081/mi.2011.e5

social phobia were presented with single faces for $10 \mathrm{~s}$ each. Both studies found that compared to healthy controls, patients had longer raw scanpaths to negative faces (a scanpath being the spatial length of the eye movement pattern), but fewer and shorter fixations to the eyes of negative faces. These effects were more pronounced, the more negatively valenced the face was (e.g. stronger for angry than for sad faces). Horley et al. ${ }^{20}$ interpreted these results as an indication that patients use strategic avoidance, especially of salient features, when confronted with threatening faces. Explicitly replicating these findings, Moukheiber et al. ${ }^{21}$ also found increased scanpaths and reduced fixations to the eyes of emotional faces, in particular those with anger and disgust expressions. Gamble and Rapee ${ }^{18}$ showed patients and controls angry, happy, and neutral faces for $5 \mathrm{~s}$. They then compared eye movements from the first $500 \mathrm{~ms}$ and the full 5 
s. While patients showed vigilance for angry faces at $500 \mathrm{~ms}$, there was no difference between groups at $5 \mathrm{~s}$. Two of the studies investigating subclinical social anxiety found support for a model of quick vigilance followed by later avoidance as proposed by Mogg et al. ${ }^{10}$ First, Garner et al. ${ }^{23}$ found that HSA students had faster initial eye movements towards the emotional face in a dot-probe task with an exposure duration of $1.5 \mathrm{~s}$ compared with LSA students. Subsequently, they fixated less on the emotional face. Second, when presented with computer generated emotional-neutral face pairs for $3 \mathrm{~s}$, Wieser, Pauli, Weyers, Alpers, and Mühlberger ${ }^{26}$ found that compared with LSA students, HSA students fixated the emotional faces longer within the first second, but during the next $500 \mathrm{~ms}$, they avoided the emotional faces.

There are a few issues with the study by Wieser et al. ${ }^{26}$ First, the two groups were formed using a median split of their scores on the Brief Fear of Negative Evaluation, ${ }^{27}$ but they did not differ on the Social Phobia and Anxiety Inventory. ${ }^{28}$ This suggests that the results may not apply to populations with more general symptoms of social anxiety. Second, computer generated faces may not be comparable to photographs of real persons. One argument against the use of artificial faces is that they do not represent someone the participants could expect to interact with or who might remind them of someone they know. One could speculate then that an artificial face may not engage socially anxious cognitions as readily as human faces. Third, the interaction between group and time was not significant $(\mathrm{P}=.08)$, while the avoidance effect was also only a trend $(\mathrm{P}=.06)$. Strictly speaking, these effects could therefore have been statistical artefacts. In any case, Wieser et al.'s ${ }^{26}$ results call for replication with photographs of real people.

The final three studies with nonclinical participants differed from the rest in their methodology. Mühlberger, Wieser, and Pauli24 had students with virtual reality headgear look at full-body avatars with happy or angry facial expressions. Either two avatars with different expressions or an avatar and a similar sized neutral object were shown. Participants' eye and head movements were continuously monitored. In this study, compared to LSA students, HSA students initially looked more often at the neutral object than the avatar, and they had fewer fixations on the avatar. Also, self-reported social anxiety correlated with an initial avoidance (within the first second) of avatars with emotional expressions, regardless of their valence. The study did not provide information as to the actual self-reported social anxiety scores in the two groups, or whether they differed significantly from each other (groups were created by median-split), thus the students in this study may not actually have been considered socially anxious outside of the experimental setting.

Buckner, Maner, and Schmidt 22 showed a face with a happy or disgusted expression alongside three non-face pictures matched with the face on dimensions of arousal and threat for $2 \mathrm{~s}$. The participants were students divided into two groups based on median-split of the scores on the Social Interaction Anxiety Scale ${ }^{29}$. Length of fixations were then analysed for four separate time intervals of $500 \mathrm{~ms}$ each. Only the last interval (from $1.5 \mathrm{~s}$ to $2 \mathrm{~s}$ ) showed a significant difference between groups in that high socially anxious participants had longer fixations to disgust faces compared with low socially anxious participants. The authors interpret this finding as evidence that social anxiety entails a difficulty in disengaging attention from threatening faces.

Finally, Wieser, Pauli, and Mühlberger25 found in an attentional control paradigm that HSA students made more errors than controls when having to deliberately move their eyes away from emotional faces presented for $1 \mathrm{~s}$. This would indicate that emotional faces distracted HSA students more than controls.

The findings from the eye tracking studies described above are not entirely consistent. While Garner et al. ${ }^{23}$ and Wieser et al. ${ }^{26}$ found initial vigilance for emotional faces, Mühlberger et al. ${ }^{24}$ found initial avoidance, and Buckner et al. ${ }^{22}$ did not find any bias. One obvious difference between these four studies is the use of full body avatars in the study by Mühlberger et al., ${ }^{24}$ while the other three used photographs of faces, and computerized faces, respectively. Finally, Horley et al. ${ }^{19-20}$ also found avoidance of photographs of faces, but they did not distinguish between initial and later fixations. Moukheiber et al.21 on the other hand did, but did not find any differences between patients and controls within the initial $300 \mathrm{~ms}$. The direction of attentional bias early in the presentation of threatening faces is therefore not very clear, with results seemingly pointing in all directions.

An interesting aspect of the studies described in the above overview is that they investigate attentional bias using very different exposure durations, from $175 \mathrm{~ms}$ in Stevens, Rist, and Gerlach, 14 over the $500 \mathrm{~ms}$ of the dot-probe, 12 to the $1.5 \mathrm{~s}$ and $3 \mathrm{~s}$ used in the studies by Garner et al. ${ }^{23}$ and Wieser et al. ${ }^{26}$ respectively, and ending with $5 \mathrm{~s}$ and $10 \mathrm{~s}$ exposures in the studies by Gamble and Rapee, ${ }^{18}$ Horley et al.,19,20 and Moukheiber et al., ${ }^{21}$ respectively. Given that different exposure durations have been used across different experimental designs, a clear picture of the time course of attentional bias is warranted. We therefore compared different exposure durations within the same experimental setting and using the same stimuli.

\section{The present study}

The primary goal of this study was to compare the findings across three different exposure durations ( $500 \mathrm{~ms}, 3 \mathrm{~s}$ and $10 \mathrm{~s}$ ) to investigate the time course of attentional bias in social anxiety. Based on existing theories and experimental results, we predicted that patients with social phobia would show a pattern of vigilance at the short exposure duration $(500 \mathrm{~ms})$ followed by avoidance at the longer exposure durations ( $2.5 \mathrm{~s}$ and $10 \mathrm{~s}$ ). Because there has been relatively few eye tracking studies with socially anxious participants, replication of prior results was a purpose in itself. First, we sought to replicate the finding by Mogg et al.17 that the bias index from the dot-probe task correlates with the direction of eye movements to the faces. As mentioned above, the reliability of the dot-probe task has previously been shown to be inadequate. ${ }^{15-16}$ Eye tracking may help to validate the basic assumption behind the task, namely that eye movements correspond to reaction time. Second we sought to replicate the finding by Wieser et al. ${ }^{26}$ that social anxiety is associated with longer initial fixations (vigilance) to the emotional face of an emotional-neutral face pair, followed by shorter fixations (avoidance) when stimuli are presented for $3 \mathrm{~s}$. Third, we wanted to replicate Horley et al.'s19-20 and Moukheiber et al.' ${ }^{21}$ findings that patients with social phobia have fewer and shorter fixations to the eye region of negative faces presented for $10 \mathrm{~s}$, and that this effect is more pronounced with angry and disgust faces over sad faces.

\section{Materials and Methods}

\section{Participants}

Eight patients (5 female, 3 male) were recruited from the Clinic for Anxiety Disorders at Aarhus University Hospital in Risskov, Denmark, and one male patient was recruited from the Anxiety Clinic at the Department of Psychology, Aarhus University, for a total of nine patients. All clinical participants were diagnosed with DSM-IV generalized social phobia by clinical psychologists using the Anxiety Disorders Interview Schedule for DSM-IV30 and participated in the study prior to entering into group therapy. Five out of nine patients had one or more comorbid diagnoses (four with depression, three with panic disorder, and one with obsessive-compulsive disorder). One female participant in the patient group did not wish to complete the procedure and was therefore excluded, leaving eight patients ( 4 male, 4 female) for the analyses.

Thirty-four control participants (18 female, 16 male) were recruited from among the Aarhus University student population. Both patients and controls were native Danish 
speakers. Table 1 shows demographic variables for both groups. The study was approved by the Central Denmark Region Committees on Biomedical Research Ethics, and all participants gave their written informed consent.

\section{Materials}

The following questionnaires were used for the study: The state form of the State-Trait Anxiety Inventory ${ }^{31}$ (STAI); Beck's Depression Inventory-II32 (BDI-II); the Brief Fear of Negative Evaluation ${ }^{27}$ (B-FNE); and the Social Interaction Anxiety Scale ${ }^{29}$ (SIAS). All questionnaires were translated into Danish using backtranslation. In the present study, Cronbach's Alpha was satisfactory (STAI: $\alpha=.966$, BDI-II: $\alpha=.890$, B-FNE: $\alpha=.701$, SIAS: $\alpha=.963)$. Nine female and 13 male models of Caucasian appearance expressing angry, happy, disgusted, and sad emotions as well as non-emotional (neutral) faces were taken from the NimStim Face Stimulus Set. ${ }^{33}$ All photos were resized to $316 \times 406$ pixels (screen size: width $=9.3 \mathrm{~cm}$, height $=12.0 \mathrm{~cm}$ ) and their backgrounds were changed to a light grey. Recording of eye movements was obtained using a Tobii X120 Eye Tracker connected to a PC running the software Tobii Studio 1.3. The X120 Eye Tracker is accurate to within $0.5^{\circ}$ visual angle and samples gaze coordinates at $120 \mathrm{~Hz}$. All trials were run on a separate PC with a 19" widescreen TFT monitor set at $1280 \times 1024$ resolution, using the software DirectRT (EmpiriSoft Corporation, New York, NY, USA).

\section{The dot-probe task}

A trial consisted of a pair of photos of the same model. Each pair was made up of either an emotional (angry or happy) and a neutral expression, or two neutral expressions. When both expressions were neutral, one had an open mouth and the other had a closed mouth to ensure that the expressions were not completely identical. The distance between the inner edges of the two faces was $11.3 \mathrm{~cm}$ corresponding to a visual angle of $9.2^{\circ}$. The trials were arranged in a fixed random order into four blocks of 36 presentations each, totalling 144 trials of which 24 were neutral-neutral pairs. During presentation, participants saw a centered, white fixation cross on a black background for $500 \mathrm{~ms}$, followed by $200 \mathrm{~ms}$ of blank screen. A pair of faces was then visible for 500 $\mathrm{ms}$ after which it was immediately replaced by a probe. The probe was a blue, 22-point bold letter (an E or an F) and participants pressed one of two keys clearly labelled $\mathrm{E}$ or $\mathrm{F}$ in response, using a customized response box (DirectIN High Speed Button-Box; EmpiriSoft Corp.) with the keys placed one above the other. The letter remained visible until participant response. In half the trials, the probe appeared on the left side of the screen and in

Table 1. Demographic and questionnaire data by group.

\begin{tabular}{lcccccc} 
& \multicolumn{2}{c}{ Social phobia } & \multicolumn{2}{c}{ Control } & & \\
& M & SD & M & SD & t & P \\
Age (range) & $29.4(22-45)$ & 9.3 & $26.2(20-54)$ & 6.7 & 1.11 & .272 \\
Percent female & 50 & & 53 & & 0.15 & .884 \\
\hline BDI-II & 20.1 & 13.2 & 5.3 & 3.9 & 2.95 & .025 \\
B-FNE & 53.6 & 5.2 & 31.5 & 8.3 & 7.19 & .000 \\
\hline SIAS & 51.3 & 11.9 & 17.0 & 10.9 & 7.87 & .000 \\
STAI & 53.8 & 14.9 & 29.0 & 6.1 & 4.59 & .002 \\
\hline
\end{tabular}

BDI-II, Beck's depression inventory-II; B-FNE, brief fear of negative evaluation; SIAS, social interaction anxiety scale; STAI, state-trait anxiety inventory, state scale.

the other half it appeared on the right side. When the letter appeared in the location of the emotional face, this was a congruent trial, and when it appeared in the location of the neutral face, it was an incongruent trial. After $500 \mathrm{~ms}$ of blank screen, the next trial commenced.

\section{Passive viewing tasks}

In the first passive viewing task, emotionalneutral face pairs were shown for $3 \mathrm{~s}$. In this task, 36 face pairs were selected to include happy and angry expressions. Every other face pair shown was a repeat from the dot-probe task (i.e. familiar), while the rest were new (i.e. unfamiliar).

In the second passive viewing task, a single enlarged face $(632 \times 812$ pixels, screen size: width $=18.6 \times$ height $=24 \mathrm{~cm}$ ) was shown at the centre of the screen for $10 \mathrm{~s}$. The face had either an angry, happy, sad, disgusted or neutral expression. Following the presentation of the face, the screen went blank for an additional $10 \mathrm{~s}$ before the next face appeared. Each of the 20 presentations was preceded by a fixation cross. Again, every other expression was a repeat from the previous tasks, while the rest were new.

\section{Procedure}

Participants arrived at the laboratory where they filled out the questionnaires and were then seated with their heads approximately 70 $\mathrm{cm}$ in front of the monitor. The eye tracker was positioned below the monitor. Participant movement was not restricted. After a short 5point calibration of the eye tracker, participants completed a computerized introduction to the dot-probe task, which included 8 trials with presentations of neutral faces only, using a model not included in the experimental tasks. They then completed the three main tasks in the following order: dot-probe task, first passive viewing task, second passive viewing task. All trials were presented against a black background. During the dot-probe task, participants were instructed to respond as quickly as possible while avoiding errors. In both of the passive viewing tasks, participants were told to simply look at the faces in any manner they chose. The researcher was seated behind a screen in order to monitor the eye tracking and make sure that participants followed instructions. Finally, the participants were debriefed and thanked for their participation. A session took approximately one hour.

\section{Data preparation}

On all trials, the Tobii Fixation filter was applied. The filter calculates fixations based on gaze coordinates that are within a region of 35 pixels $\left(0.8^{\circ}\right.$ visual angle in this study). For the first passive viewing task, the faces themselves and their immediate background were considered areas of interest. For the second passive viewing task, two areas of interest were created individually for each face covering each eye (mean size: height $=2.5 \mathrm{~cm}$, length $=3.6 \mathrm{~cm}$ ). Importantly, since the eye tracker showed some measurement error for a majority of the participants, the areas of interest were manually adjusted to reflect this discrepancy. This was achieved by measuring the mean distance from a participant's fixations to the fixation cross and then moving the area of interest correspondingly (mean $2.5 \mathrm{~cm}$ horizontal and $0.2 \mathrm{~cm}$ vertical). An independent rater completed the procedure for a subset of participants $(n=14)$ and the inter-rater agreement was satisfactory $(r=.94$ for horizontal adjustment and $r=.89$ for vertical adjustment).

\section{Statistical analyses}

Data were analyzed using SPSS 17. Missing items in the questionnaires $(\mathrm{k}=2)$ were replaced using the mean substitution function in SPSS, whereas errors on the dot-probe task (2.2\%) were deleted. One male participant in the control group had $19.4 \%$ errors on the dotprobe task and his data were removed from this analysis. For each trial in the dot-probe task, if a participant's reaction time was identified as an outlier by SPSS in relation to his/her own average, it was changed to $1 \mathrm{~ms}$ higher or lower than the second-most extreme reaction time for that participant following recommendations by Tabachnick and Fidell. ${ }^{34}$ Such outliers accounted for $2.4 \%$ of the data. In the two passive viewing tasks, data was deleted from trials with no eye tracking data, and missing values accounted for $0 \%$ and $2.6 \%$ of 
the data, respectively. Outliers were changed following the same procedure as above and accounted for $4.8 \%$ in the first passive viewing task and $2.7 \%$ in the second passive viewing task. One male participant in the control group made only very few eye movements on the three tasks, while equipment malfunction meant that eye tracking data was not available for one additional male and one female participant in the control group. Thus, these three participants were excluded from the eye tracking analyses, but their reaction time scores on the dot-probe task were retained.

In the dot-probe task a bias index was calculated by subtracting the reaction time to congruent trials from reaction time to incongruent trials and dividing the result by two. ${ }^{11}$ Positive scores on this bias index indicate vigilance for the emotional face, while negative scores indicate avoidance. The bias indices were then entered into a $2 \times 2$ repeated measures ANOVA with group as a between subject factor and valence (angry, happy) as a within subject factor. Furthermore, for each trial the direction of the last saccade preceding offset of the faces was determined. The last saccade was defined as the last recorded eye movement to enter or leave the location of either face, while they were still fully visible on the screen. Following Mogg et al.,17 a bias score was calculated for each participant by counting the number of trials with an eye movement towards the emotional face proportional to the total number of trials for that expression. For example, the bias score for angry faces would be the total number of trials with angry-neutral face pairs divided by the number of trials where the participant's last eye movement was toward the angry face. A bias score above 0.5 indicates vigilance for the angry face, while a score below 0.5 indicates avoidance. Thus, bias index refers to reaction time data, while bias score refers to eye movements.

In the first passive viewing task, time to first fixation, total fixation count, and total fixation duration was calculated separately for the first $500 \mathrm{~ms}$ and the last $2.5 \mathrm{~s}$ of the $3 \mathrm{~s}$ display. These data were entered into separate $2 \times 2 \times 2$ repeated measures ANOVAs with group as a between subject factor and time (first $500 \mathrm{~ms}$ vs. last $2.5 \mathrm{~s}$ ) and valence (angry, happy) as within subject factors. In the second passive viewing task, time to first fixation, total number of fixations, and fixation duration to the eye region of the faces were entered into separate $2 \times 5$ repeated measures ANOVAs with group as a between subject factor and valence of faces (happy, disgust, angry, sad, neutral) as a within subject factor.

\section{Results}

\section{Questionnaires}

Patients and controls did not differ on age or gender, but they differed significantly on all questionnaire measures, with patients having higher scores of social anxiety (B-FNE, SIAS), depression (BDI-II) and state anxiety (STAI) (Table 1).

\section{Dot-probe task}

The main effect of group and the interaction between group and valence were not significant (P's >.05). However, there was a significant main effect of valence $(F(1.39)=4.60$, $\mathrm{P}=.038$ ). One-sample t-tests showed that this main effect was due to participants showing no bias to the angry faces $(\mathrm{P}>.05)$ and vigilance for happy faces $(\mathrm{t}(40)=4.84, \mathrm{P}<.001)$. Turning to the eye movement analysis, only participants with at least $50 \%$ valid trials were included $(n=32)$ due to a high number of missing values (e.g. loss of eye tracking data, lack of eye movements, and eye movements outside the areas of interest). The bias score and the bias index correlated significantly for happy faces $(\mathrm{r}=.459, \mathrm{P}=.007)$ and for angry faces $(\mathrm{r}=.405, \mathrm{P}=.019) . \mathrm{A} 2 \times 2$ repeated measures ANOVA with group as a between subject factor and valence as within subject factor did not show any significant effects (all P's<.05).

\section{First passive viewing task ( $3 \mathrm{~s}$ )}

Time to first fixation showed a main effect of valence $(\mathrm{F}(1.37)=17.12, \mathrm{P}<.001)$, but there were no effects including group. One-sample ttests revealed that participants showed avoidance of happy faces at $500 \mathrm{~ms}(\mathrm{t}(38)=4.56$, $\mathrm{P}<.001)$ and vigilance for angry faces at 500 $\mathrm{ms}(\mathrm{t}(38)=2.45, \mathrm{P}=.019)$.

Total fixation count showed a main effect of valence $(\mathrm{F}(1.37)=10.43, \mathrm{P}=.003)$ and group $(\mathrm{F}(1.37)=12.89, \mathrm{P}=.001)$ and a group $\mathrm{x}$ time interaction $(\mathrm{F}(1.37)=7.64, \mathrm{P}=.009)$. The main effect of valence was due to all participants showing vigilance for happy faces at $500 \mathrm{~ms}$ $(\mathrm{t}(38)=3.68, \mathrm{P}=.001)$ and $2.5 \mathrm{~s}(\mathrm{t}(38)=4.09$, $\mathrm{P}<.001)$, and avoidance of angry faces at 500 $\mathrm{ms}(\mathrm{t}(38)=2.89, \mathrm{P}=.006)$ with no bias at $2.5 \mathrm{~s}$ $(\mathrm{P}<.05)$. Investigating the group $\mathrm{x}$ time interaction, we found that patients had more fixations to happy faces compared with controls at both $500 \mathrm{~ms}(\mathrm{t}(24.0)=4.21, \mathrm{P}<.001)$ and $2.5 \mathrm{~s}$ $(\mathrm{t}(37)=3.42, \mathrm{P}=.002)$, while there was no difference between groups at $500 \mathrm{~ms}$ for the angry faces $(\mathrm{P}<.05)$. At $2.5 \mathrm{~s}$, patients had more fixations to angry faces compared with controls $(\mathrm{t}(37)=2.21, \mathrm{P}=.034)$.

Total fixation duration showed a main effect of valence $(\mathrm{F}(1.37)=27.13, \mathrm{P}<.001)$ and $\mathrm{a}$ valence $\mathrm{x}$ time interaction $(\mathrm{F}(1.37)=16.75$, $\mathrm{P}<.001)$. All participants showed vigilance for happy faces at both $500 \mathrm{~ms}(\mathrm{t}(38)=2.05$, $\mathrm{P}=.047)$ and $2.5 \mathrm{~s}(\mathrm{t}(38)=7.65, \mathrm{P}<.001)$, while they showed avoidance of angry faces at 500 $\mathrm{ms}(\mathrm{t}(38)=2.42, \mathrm{P}=.020)$. At $2.5 \mathrm{~s}$, there was no difference in fixation duration between happy and angry faces $(\mathrm{P}>$.05). The two groups did not differ in the length of their fixations to the faces.

\section{Second passive viewing task (10 s)}

Time to first fixation showed a main effect of valence $(\mathrm{F}(4.31)=3.89, \mathrm{P}=.011)$, with no effects of group reaching significance. To further examine the effect of valence, we used paired-samples t-tests to compare results for negative faces with results for the happy and neutral faces. The mean for angry faces differed significantly from the mean for happy $(\mathrm{t}(36)=2.28, \mathrm{P}=.029)$ and neutral faces $(\mathrm{t}(36)$ $=2.65, \mathrm{P}=.012)$, and the mean for disgust faces also differed significantly from the mean for happy $(\mathrm{t}(37)=2.15, \mathrm{P}=.038)$ and neutral faces $(\mathrm{t}(37)=2.44, \mathrm{P}=.019)$. Sad faces did not differ from neutral or happy faces. Inspection of means showed that all participants were slower to fixate the eyes of angry and disgust faces, indicating avoidance. Total fixation count also showed a main effect of valence $(\mathrm{F}(4.32)=$ $5.48, \mathrm{P}=.002)$ while no other effects were significant. Paired-samples t-tests showed that the total number of fixations to the eyes of angry faces was significantly lower than for happy $(\mathrm{t}(37)=3.10, \mathrm{P}=.004)$ and neutral faces $(\mathrm{t}(37)=2.36, \mathrm{P}=.024)$. Also the mean for disgust faces was significantly lower than for happy $(\mathrm{t}(36)=4.66, \mathrm{P}<.001)$ and neutral faces $(\mathrm{t}(36)=5.84, \mathrm{P}<.001)$. Sad faces did not differ from either happy or neutral faces $(\mathrm{P}>.05)$.

Finally, the total duration of the fixations to the eyes of faces showed a significant main effect of valence $(\mathrm{F}(4.32)=5.72, \mathrm{P}=.001)$ and a main effect of group $(\mathrm{F}(1.35)=5.92, \mathrm{P}=.020)$ with no other effects being significant. Pairedsamples t-tests showed that the effect of valence was due to the mean fixation duration for disgust faces to be significantly shorter than for happy $(\mathrm{t}(36)=5.30, \mathrm{P}<.001)$ and neutral faces $(\mathrm{t}(36)=5.46, \mathrm{P}<.001)$. Angry and sad faces did not differ from neutral or happy expressions. Furthermore, the main effect of group was due to patients having shorter fixation durations to the eyes of neutral $(\mathrm{t}(36)=$ 2.33, $\mathrm{P}=.026)$, sad $(\mathrm{t}(23.8)=3.98, \mathrm{P}=.001)$, and disgust faces $(\mathrm{t}(31.7)=2.60, \mathrm{P}=.014)$.

\section{Correlations with questionnaires}

In the dot-probe task, the reaction time bias index to happy faces correlated negatively with fear of negative evaluation (B-FNE; $r=-.326$, $\mathrm{P}=.037$ ) and state anxiety (STAI; $\mathrm{r}=-.444$, $\mathrm{P}=.004$ ), but not with depression (BDI-II) or social interaction anxiety (SIAS). The bias index to angry faces did not show any significant correlations at the .05 level (range: $r=$ 
.045 to $\mathrm{r}=.187$ ). Eye movements on the dotprobe task also did not show any significant correlations with questionnaires (range: $r=-$ .271 to $\mathrm{r}=.336$, all P's $>.05$ ). There was a tendency however, for a significant correlation between vigilance for angry faces and social interaction anxiety ( $\mathrm{r}=.336, \mathrm{P}=.07)$.

In the first passive viewing task, none of the correlations between fixation duration to angry, happy or neutral faces correlated significantly with questionnaire scores at the .05 level (range: $r=-.265$ to $r=.236$ ). In the second passive viewing task, state anxiety correlated with shorter fixations to the eyes of sad and neutral faces (sad: $\mathrm{r}=.425, \mathrm{P}=.008$; neutral: $\mathrm{r}=.348, \mathrm{P}=.032$ ). Both social anxiety measures also correlated with shorter fixations to the eyes of sad faces (B-FNE: $\mathrm{r}=.330, \mathrm{P}=.043$; SIAS: $\mathrm{r}=.325, \mathrm{P}=.046)$. No other correlations were significant (range: $\mathrm{r}=-.315$ to $\mathrm{r}=.210, \mathrm{P}>.05$ ), but both state anxiety and fear of negative evaluation tended to correlate with avoidance of the eyes of happy faces (STAI: $r=-.302$, $\mathrm{P}=.07$; B-FNE: $\mathrm{r}=-.315, \mathrm{P}=.05$ ).

\section{Discussion}

The main findings across the three different tasks are summarized in Table 2. We found significant effects of group in all tasks and at all three time points investigated, but contrary to our hypotheses, the results did not unequivocally support the vigilance-avoidance theory of attentional bias. The results of each task are discussed below.

The bias index based on reaction time correlated significantly with bias scores based on eye movements. This finding validates the assumption underlying the dot-probe task that the direction in eye movements is directly related to reaction time as first reported by Mogg et al. ${ }^{17}$ We did not find that the patients differed from controls in attentional bias to angry and happy faces on the dot-probe task and also did not find any attentional bias when analyzing eye movements. Since the overall effect size of the dot-probe task has been estimated to be around $d=0.3735$, the current sample size may have been too low to find any significant effects. A post-hoc power analysis revealed that our statistical power to detect the difference for angry faces was .36 (for Cohen's $d=0.15$ ), while for happy faces it was .99 (for Cohen's $d=0.52$ ). This indicates that the current study did not have sufficient statistical power to detect a difference in attentional bias to angry faces between patients and controls on the dot-probe task, while the non-significant result for happy faces appears much more robust.

In the first passive viewing task, compared to controls, patients had more fixations to

Table 2. Summary of attentional bias by task and by group.

$\begin{array}{lll} & \text { Social phobia } & \text { Control } \\ \text { Task Expression } & \text { within group Between groups }\end{array}$ Within group

\begin{tabular}{|c|c|c|c|c|}
\hline Dot-probe $500 \mathrm{~ms}$ & $\begin{array}{l}\text { Angry } \\
\text { Happy }\end{array}$ & $\begin{array}{l}\text { No bias } \\
\text { Vigilance }\end{array}$ & & $\begin{array}{l}\text { No bias } \\
\text { Vigilance }\end{array}$ \\
\hline Passive viewing $500 \mathrm{~ms}$ & $\begin{array}{l}\text { Angry } \\
\text { Happy }\end{array}$ & $\begin{array}{l}\text { Vigilance* } \\
\text { Avoidance } \\
\text { Avoidance* } \\
\text { Vigilance }^{\text {\# }}\end{array}$ & $\mathrm{SP}>$ control $^{\circ}$ & $\begin{array}{l}\text { Vigilance* } \\
\text { Avoidance } \\
\text { Avoidance* } \\
\text { Vigilance }^{\text {\# }}\end{array}$ \\
\hline Passive viewing $2.5 \mathrm{~s}$ & $\begin{array}{l}\text { Angry } \\
\text { Happy }\end{array}$ & $\begin{array}{l}\text { No bias }{ }^{\circ} \\
\text { Vigilance }^{\text {\# }}\end{array}$ & $\begin{array}{l}\mathrm{SP}>\text { control }^{\circ} \\
\mathrm{SP}>\text { control }^{\circ}\end{array}$ & $\begin{array}{l}\text { No bias }{ }^{\circ \#} \\
\text { Vigilance }^{\circ \#}\end{array}$ \\
\hline Passive viewing $10 \mathrm{~s}$ & $\begin{array}{l}\text { Angry } \\
\text { Happy } \\
\text { Sad } \\
\text { Disgust } \\
\text { Neutral }\end{array}$ & $\begin{array}{l}\text { Avoidance*० } \\
\text { No bias } \\
\text { No bias } \\
\text { Avoidance*\# } \\
\text { No bias }\end{array}$ & $\begin{array}{l}\mathrm{SP}<\text { control }^{\#} \\
\mathrm{SP}<\text { control }^{\#} \\
\mathrm{SP}<\text { control }^{\#}\end{array}$ & $\begin{array}{l}\text { Avoidance* } \\
\text { No bias } \\
\text { No bias } \\
\text { Avoidance*\# } \\
\text { No bias }\end{array}$ \\
\hline
\end{tabular}

SP, social phobia; < indicates greater avoidance; > indicates greater vigilance; *time to first fixation; ${ }^{\circ}$ total fixation count; \#total fixation duration

happy faces during both the $500 \mathrm{~ms}$ and the 2.5 $\mathrm{s}$ intervals and to angry faces during the $2.5 \mathrm{~s}$ interval, but not to angry faces during the initial $500 \mathrm{~ms}$. Thus, patients avoided fixating the angry faces initially, while subsequently fixating them more often than controls. This result appears to be in direct contrast with the vigilance-avoidance hypothesis. However, all participants were quicker to look at the angry faces during the initial $500 \mathrm{~ms}$, while fixating them less within this same time interval. This is consistent with the vigilance-avoidance theory, but at a much shorter time scale than what we expected, and independent of social anxiety. One possible explanation is that these attentional processes happen at much shorter durations than $500 \mathrm{~ms}$ as is also suggested by some dot-probe studies with very brief exposure durations. ${ }^{36}$ However, in a recent study by Stevens et al.,14 using an electrooculogram, participants were found to make very few eye movements when exposed to faces for $175 \mathrm{~ms}$ indicating that any effects of the stimuli may be due to covert rather than overt attention.

The results for the first passive viewing task did not replicate Wieser et al., ${ }^{26}$ who found a pattern of early vigilance followed by later avoidance to both happy and angry faces in HSA participants. Some important differences between the two studies may explain these discrepancies. First, Wieser et al. used artificial computer-generated faces as stimuli, while we used photographs of people, and it is possible that artificial and real faces produce different responses. Second, the clinical participants in our study had substantially higher scores on the B-FNE than the HSA participants in Wieser et al.'s study, possibly leading to differences in attentional bias $(M=53.6, \mathrm{SD}=5.2$ versus $M=$ $44.1, \mathrm{SD}=11.2$ ). One could speculate that subclinical social anxiety leads to attentional vigi- lance for threatening faces, while clinical social anxiety may lead to avoidance due to a higher level of perceived threat. Future studies could investigate this hypothesis by comparing participants selected for subclinical social anxiety with clinically anxious participants.

In the second passive viewing task at $10 \mathrm{~s}$, patients with social phobia had shorter fixation durations to the eyes of neutral, sad, and disgust faces compared to controls. These results replicate and extend the findings by Horley et al. ${ }^{19}$ that participants with social phobia made fewer fixations to the eyes of neutral and sad faces compared with controls. There were no differences between groups in fixation duration to the eyes of angry or happy faces, but we did find a tendency for participants with social phobia to also avoid the eyes of these faces compared to controls (independent t-test for fixation duration, angry: $\mathrm{t}(37)=1.70, \quad \mathrm{P}=.094 ; \quad$ happy: $\mathrm{t}(36)=1.84$, $\mathrm{P}=.074)$. It is therefore possible that these differences would have been significant in a larger sample. Although all participants in our study took longer to fixate the eyes of angry faces and had fewer fixations to them, they did not have shorter fixations to the eyes of the angry faces. One possible explanation for this result is the disengagement difficulty theory proposed by Fox and colleagues.37 The theory states that once high anxious individuals attend to a threatening cue, disengaging attention from the cue again will be difficult, thus increasing the fixation duration. Although we found longer fixations to the eyes of angry faces relative to the number of fixations to them - consistent with a difficulty to disengage attention from threat - we did not observe any effects of social anxiety on this parameter. Finally, there were only few correlations with questionnaire scores throughout 
the three tasks, but they were all consistent with our expectations. Briefly, at $500 \mathrm{~ms}$ exposure duration, social anxiety and state anxiety correlated negatively with vigilance for happy faces, while these measures tended to correlate with vigilance for angry faces. At $10 \mathrm{~s}$ exposure duration, social anxiety and state anxiety correlated with avoidance of the eyes of sad faces with a tendency towards significance for avoidance of the eyes of happy faces as well. These results indicate that being highly anxious during the experiment led participants to avoid both positive and negative emotions at the longest exposure duration, while showing a tendency to attend to briefly presented angry faces.

\section{Limitations}

The low number of clinical participants increased the risk of type-2 error in the current study, which was especially problematic for the interpretation of near-significant effects. It is possible that avoidance of the eyes of angry and happy faces in the second passive viewing task would have reached significance in a larger sample. Also, some of the correlations between eye movements to emotional faces and questionnaire scores might have been significant in a larger sample. The small sample size also increased the risk of sampling bias. In other words, we can not be certain that the patients included in the study were representative for the general population of individuals suffering from social phobia. For these reasons, the present results should be interpreted with some caution. Secondly, the design was not counterbalanced, so all participants completed an identical procedure. We were therefore unable to control for order effects, such as habituation to the expressions. Finally, even though social phobia was the primary diagnosis in the patient group, we can not be certain that comorbid diagnoses did not influence the results. However, scores on the BDI-II did not correlate with any of the effects in the present study, reducing the likelihood that depression could account for the effects.

\section{Emotion processing in psy- chopathology}

Deficits in processing of emotional human faces have risen to be a defining feature of a range of psychiatric disorders over the past decades. Hyperactivation of the amygdala in response to negative facial expressions has been found not only in social phobia, but also in specific phobia, post-traumatic stress disorder, borderline personality disorder, and depression. ${ }^{38-40}$ Healthy adults typically also show amygdaloid activation to threatening faces, but to a lesser extent than patients. ${ }^{39,41}$ This would suggest a continuum, where higher levels of limbic activation lead to an exag- gerated fear response to threatening faces (i.e., attentional bias). However, some studies indicate that the picture may not be as straightforward: Gur et al. ${ }^{41}$ and Blair et al. ${ }^{42}$ found a hypoactive limbic response to threatening faces in patients with generalized anxiety disorder (GAD) and patients with schizophrenia, respectively. One possible moderator of limbic activation might be efficiency of regulation from frontal areas such as the anterior cingulated cortex or the ventromedial prefrontal cortex. ${ }^{39}$ For example, patients with GAD who show reduced amygdala activation to fearful faces, also show increased activation of the lateral part of the middle frontal gyrus. ${ }^{42} \mathrm{An}$ important avenue for future research is to further examine the relationship between activation of cortical and limbic structures and attentional vigilance and avoidance.

\section{Conclusions}

The present results add to a growing literature of eye tracking studies in socially anxious individuals. Contrary to our hypotheses, we did not find unequivocal support for the vigilanceavoidance theory of attentional bias proposed by Mogg et al. 10 First, patients did not show avoidance of angry faces on the dot-probe task compared to controls. Secondly, when looking at the eye tracking data, patients avoided angry faces initially, but subsequently fixated them more than controls. We did find a vigilanceavoidance pattern of attentional bias within the first $500 \mathrm{~ms}$ for all participants regardless of group, which indicates that these processes may operate faster than we expected. Finally, at the longest time interval patients showed more avoidance of the eyes of neutral, sad, and disgust faces compared with controls.

Attention to environmental threat has been proposed as an important maintaining or causal factor in social anxiety. The present study demonstrated that patients with social phobia show a complex pattern of early vigilance-avoidance for threatening faces followed by later avoidance of the eyes of negative and neutral faces. Neuroimaging studies suggest that this pattern may be due to limbic hyperactivity and possibly also inefficient regulatory processes in the frontal cortex. Interventions directed at attentional processes, such as attention training paradigms ${ }^{43}$ may prove to be valuable supplementary therapeutic tools in the treatment of anxiety disorders.

\section{References}

1. Calvo MG, Nummenmaa L. Detection of emotional faces: Salient physical features guide effective visual search. J Exp Psychol Gen 2008;137:471-94.

2. Frischen A, Bayliss AP, Tipper SP. Gaze cuing of attention: Visual attention, social cognition, and individual differences. Psychol Bull 2007;133:694-724.

3. Dimberg U, Öhman A. Behold the wrath: Psychophysiological responses to facial stimuli. Motiv Emotion 1996;20:149-82.

4. Eimer M, Holmes A. Event-related brain potential correlates of emotional face processing. Neuropsychologia 2007;45:15-31.

5. Adolphs R, Gosselin F, Buchanan TW, et al. A mechanism for impaired fear recognition after amygdala damage. Nature 2005;433:68-72.

6. Adolphs R, Tranel D, Damasio H, Damasio AR. Impaired recognition of emotion in facial expressions following bilateral damage to the human amygdala. Nature 1994;372:669-72.

7. Staugaard SR. Threatening faces and social anxiety: A literature review. Clin Psychol Rev 2010;30:669-90.

8. Clark DM, Wells A. A cognitive model of social phobia. In: Heimberg RG, Liebowitz MR, Hope DA, Schneier FR, Eds. Social phobia: Diagnosis, assessment, and treatment. New York, NY: Guilford Press; 1995. pp. 69-93.

9. Rapee RM, Heimberg RG. A cognitivebehavioral model of anxiety in social phobia. Behav Res Ther 1997;35:741-56.

10. Mogg K, Bradley BP, de Bono J, Painter M. Time course of attentional bias for threat information in non-clinical anxiety. Behav Res Ther 1997;35:297-303.

11. Chen YP, Ehlers A, Clark DM, Mansell W. Patients with generalized social phobia direct their attention away from faces. Behav Res Ther 2002;40:677-87.

12. Sposari JA, Rapee RM. Attentional bias toward facial stimuli under conditions of social threat in socially phobic and nonclinical participants. Cogn Ther Res 2007;31:23-37.

13. Stevens S, Rist F, Gerlach AL. Influence of alcohol on the processing of emotional facial expressions in individuals with social phobia. Brit J Clin Psychol 2009;48:125-40.

14. Stevens S, Rist F, Gerlach AL. Eye movement assessment in individuals with social phobia: Differential usefulness for varying presentation times? J Behav Ther Exp Psy 2011;42:219-24.

15. Schmukle SC. Unreliability of the dotprobe task. Eur J Personality 2005;19:595605.

16. Staugaard SR. Reliability of two versions of the dot-probe task using photographic faces. Psychol Sci Q 2009;51:339-50.

17. Mogg K, Garner M, Bradley BP. Anxiety and orienting of gaze to angry and fearful 
faces. Biol Psychol 2007;76:163-69.

18. Gamble A, Rapee R. The time-course of attention to emotional faces in social phobia. J Behav Ther Exp Psy 2010;41:39-44.

19. Horley K, Williams LM, Gonsalvez C, Gordon E. Social phobics do not see eye to eye: A visual scanpath study of emotional expression processing. J Anxiety Disord 2003;17:33-44.

20. Horley K, Williams LM, Gonsalvez C, Gordon E. Face to face: Visual scanpath evidence for abnormal processing of facial expressions in social phobia. Psychiat Res 2004;127:43-53.

21. Moukheiber A, Rautureau G, Perez-Diaz F, et al. Gaze avoidance in social phobia: Objective measure and correlates. Behav Res Ther 2010;48:147-51.

22. Buckner JD, Maner JK, Schmidt NB. Difficulty disengaging attention from social threat in social anxiety. Cogn Ther Res 2010;34:99-105.

23. Garner M, Mogg K, Bradley BP. Orienting and maintenance of gaze to facial expressions in social anxiety. J Abnorm Psychol 2006;115:760-70.

24. Mühlberger A, Wieser MJ, Pauli P. Visual attention during virtual social situations depends on social anxiety. CyberPsychol Behav 2008;11:425-30.

25. Wieser MJ, Pauli P, Mühlberger A. Probing the attentional control theory in social anxiety: An emotional saccade task. CABN 2009;9:314-22.

26. Wieser MJ, Pauli P, Weyers P, Alpers GW, Mühlberger A. Fear of negative evaluation and the hypervigilance-avoidance hypoth- esis: An eye-tracking study. J Neural Trans 2009;116:717-23.

27. Leary MR. A brief version of the Fear of Negative Evaluation Scale. Pers Soc Psychol B 1983;9:371-75.

28. Turner SM, Beidel DC, Dancu CV, Stanley MA. An empirically derived inventory to measure social fears and anxiety: the Social Phobia and Anxiety Inventory (SPAI). J Consult Clin Psychol 1989;1:3540.

29. Mattick RP, Clarke JC. Development and validation of measures of social phobia scrutiny fear and social interaction anxiety. Behav Res Ther 1998;36:455-70.

30. Brown TA, DiNardo PA, Barlow DH. Anxiety disorders interview schedule for ADIS-IV. Albany, NY: Graywind Publications Incorporated; 1994.

31. Spielberger CD, Gorsuch RL, Luschene RE, et al. Manual for the State-Trait Anxiety Inventory. New York, NY: Consulting Psychologists Press; 1983.

32. Beck AT, Steer RA, Brown GK. Manual for the Beck Depression Inventory, 2nd ed. San Antonio, TX: The Psychological Corporation; 1996.

33. Tottenham N, Tanaka J, Leon AC, et al. The NimStim set of facial expressions: Judgments from untrained research participants. Psychiat Res 2009;168:242-49.

34. Tabachnick BG, Fidell LS. Using Multivariate Statistics. 4th Ed. Boston: Allyn \& Bacon; 2001.

35. Bar-Haim Y, Lamy D, Pergamin L, et al. Threat-related attentional bias in anxious and non-anxious individuals: A meta-ana- lytic study. Psychol Bull 2007;133:1-24.

36. Cooper RM, Langton SRH. Attentional bias to angry faces using the dot-probe task? It depends when you look for it. Behav Res Ther 2006;44:1321-29.

37. Fox E, Russo R, Bowles R, Dutton K. Do threatening stimuli draw or hold visual attention in subclinical anxiety? J Exp Psychol Gen 2001;130:681-700.

38. Domes G, Schulze L, Herpertz SC. Emotion recognition in borderline personality disorder $-\mathrm{a}$ review of the literature. J Pers Disord 2009;23:6-19.

39. Etkin A, Wager T. Functional neuroimaging of anxiety: A meta-analysis of emotional processing in PTSD, social anxiety disorder, and specific phobia. Am J Psychiat 2007;164:1476-88.

40. Leppänen JM. Emotional information processing in mood disorders: A review of behavioral and neuroimaging findings. Curr Opin Psychiatr 2006;19:34-9.

41. Gur RE, McGrath C, Chan RM, et al. An fMRI study of facial emotion processing in patients with schizophrenia. Am J Psychiat 2002;159:1992-99.

42. Blair K, Shaywitz J, Smith BW, et al. Response to emotional expressions in generalized social phobia and generalized anxiety disorder: Evidence for separate disorders. Am J Psychiat 2008;165:1193202.

43. Schmidt NB, Richey JA, Buckner JD, Timpano KR. Attention training for generalized social anxiety disorder. J Abnorm Psychol 2009;118:5-14. 